\title{
Special Features of Historical Papers from the Viewpoint of Bibliometrics
}

\author{
Werner Marx \\ Max Planck Institute for Solid State Research, Heisenbergstraße 1, D-70569 Stuttgart, Germany. \\ E-mail:W.Marx@fkf.mpg.de
}

This paper deals with the specific features of historical papers relevant for information retrieval and bibliometrics. The analysis is based mainly on the citation indexes accessible under the Web of Science (WoS) but also on field-specific databases: the Chemical Abstracts Service (CAS) literature database and the INSPEC database. First, the journal coverage of the WoS (in particular of the WoS Century of Science archive), the limitations of specific search fields as well as several database errors are discussed. Then, the problem of misspelled citations and their "mutations" is demonstrated by a few typical examples. Complex author names, complicated journal names, and other sources of errors that result from prior citation practice are further issues. Finally, some basic phenomena limiting the meaning of citation counts of historical papers are presented and explained.

\section{Introduction}

Some field-specific literature databases, like the Chemical Abstracts Service (CAS) literature database and the INSPEC database for physics, electronics, and computing, now extend coverage back to 1900 (or even some years before 1900; see http://www.stn-international.de/stndatabases/databases/). The launching of the Century of Science backfiles by Thomson Reuters (see http://wokinfo.com/products_tools/ multidisciplinary/webofscience/ and http://wokinfo.com/ products_tools/backfiles/cos/) extended the retrospective coverage of Web of Science (WoS) back to 1900:

This has made it possible to comprehensively analyze the citations to historic works of the early part of the 20th century, a so called golden era for scientific discovery, especially in the field of theoretical chemistry and physics. There are several very highly cited works in this period, and many of these are being more heavily cited today than at any time in their history. (Pratt \& Stembridge, 2006, p. 2)

The Scopus database, produced by Elsevier (see http:// www.info.sciverse.com/scopus/), is increasingly covering

Received December 7, 2010; revised December 13, 2010; accepted December 13, 2010

(C) 2011 ASIS\&T • Published online 25 January 2011 in Wiley Online Library (wileyonlinelibrary.com). DOI: 10.1002/asi.21479 earlier literature; however, at present, Scopus counts citations only since 1996. Hence, Scopus is currently less important with respect to bibliometrics of historical papers.

The availability of the bibliographic data (including the citation counts) of papers ${ }^{1}$ published in the first half of the 20th century has two main aspects: First, the data satisfy the needs of researchers who like to refer to early papers and build upon that work. In addition, the data ought to be of potential interest for bibliometric studies in the field of the history of science. However, there are caveats to searching historical papers and counting their citations as a measure of their impact: the limited coverage of journals, limitations of specific search fields, database errors, translation errors, problems arising from misspelled citations, complex author names, complicated journal names, and other sources of errors resulting from the citation practice in the past. Finally, in the case of historical papers there are some basic phenomena limiting the meaning of citation counts as a measure of scientific impact.

\section{Limited Journal Coverage}

At the time when WoS was extended back to 1900 in the Century of Science project, not all relevant material was available and could be included. For example, some volumes of the prestigious journal Philosophical Magazine are missing in WoS for reasons unknown: 1900 (Vol. 50), 1903 (Vol. 6), 1904 (Vol. 7), 1911 (Vol. 21), 1915 (Vol. 29), and possibly more. The volumes of the publication years 1913, 1945-1948, and 1950 are completely missing. There are also gaps in coverage of many other journals reaching back to the first half of the 20th century. However, Thomson Reuters kindly makes available on request detailed lists of their journal coverage that reveal all gaps in coverage of the specific journals. This means that WoS users do not need to explore the coverage of a given journal by themselves. Further, some journals have not been included from the time of their inception (or since

\footnotetext{
${ }^{1}$ To avoid confusion with the WoS document type "article," the term "paper" rather than "article" is used here for any kind of journal-based publication.
} 
1900) in the ensemble of the WoS source journals (the approximately 11,000 journals currently selected as contributing significantly to the progress of science). For example, the journal Angewandte Chemie, now one of the most prestigious journals in the field of chemistry, was founded in the year 1888 but is not covered by WoS prior to 1932 .

The gaps in coverage may also have political reasons. For example, missing in WoS are all volumes in the publication year 1950 of the translated version of the Russian journal Soviet Physics JETP (original name: Zhurnal Eksperimentalnoi i Teoreticheskoi Fiziki, ZhETF). A possible explanation is given in the note added in proof of the paper by Cardona and Marx (2006a, 2009):

\begin{abstract}
After the manuscript was completed, A. Wittlin pointed out to us the possible reason why the complete 1950 issues of the ZhETF are missing in the Western literature databases. This reason can be found in the closing chapter of R. D. Parks's book on superconductivity (Marcel Dekker, New York, Vol. 2, p. 1347, 1969), written by P.W. Anderson. The year 1950 marks the early days of the Cold War as well as the concomitant beginning of McCarthyism in the USA. In those witch-hunting days, Soviet publications, including scientific journals, were either formally or informally banned. Anderson suggests that issues arriving to the USA may have been dumped straight into the harbors.
\end{abstract}

\section{Limitations of Search Fields}

The accessibility of early literature is a question of not only journal coverage but also data availability within specific search fields offered by the relevant databases. Some examples will demonstrate this: In contrast to the field-specific literature databases, there are no abstracts available before 1991 in the citation indexes accessible in WoS, with serious limitations for searching topics. Topic searching is limited to the selection of title words only. Authors' addresses including country are predominantly missing in the WoS databases before 1973. Therefore, address-specific searches, such as the distribution of papers throughout certain countries or institutions, are not possible for the pre-1973 literature. As for earlier literature, the lack of addresses is a problem not only of the WoS citation indexes but also of the field-specific literature databases. The INSPEC database does not provide address information before around 1968 and the CAS literature database at least not before around 1950. The lack of address-specific information, in particular before 1950, is mainly caused by the poor coverage of this kind of information by journals at that time. Note that in contrast to WoS, most field-specific literature databases like the CAS and the INSPEC database do not include the addresses of the coauthors but only either the address of the first author or the corresponding author of a given paper.

\section{Database Errors}

Database errors are introduced in the construction of the databases and are an issue with regard to historical work.
Many journals that stretch throughout a century or more have a complex history and highly specific features that possibly contradict database standards. Sometimes it is too difficult for the database producers to adjust their databases to the needs presented by such journals and to clean the data by correcting errors. This is illustrated by some examples of typical database errors below:

The journal Angewandte Chemie has been published since 1962 in both a German and an international edition, which has led to a number of problems with respect to citation analyses (Marx, 2001). Because Angewandte Chemie papers are contained in different volumes and appear on different page numbers in the two editions, these papers appear as if they were two completely different publications. This is a source of error for both the citation counts of single papers and the journal impact factor as a measure of the performance of the journal as a whole. For the time period from 1932 (the first year of coverage) to 1955 , WoS considered the numerical source data (in particular volume number and pagination) of the German edition and correctly assigned the records to German as the original language. After 1955, the bibliographic data of the German edition are continued, but in the journal name field the international edition appears. From 1962 onwards, the year of the inception of the international edition, the journal name, and the numerical source data again fit together.

A similar error of assignment with regard to the original language happened with Zeitschrift für Metallkunde (since 2006: International Journal of Materials Research): The pre1969 papers in this journal are assigned incorrectly. For the time period 1948-1968, WoS misleadingly assigned English instead of German as the original language, curiously with the exception of publication year 1964 (Marx, 2009). This error is possibly caused by the fact that between 1956 and around 1970, the papers published in German additionally included a summary in English. We may assume that there are other journals where the original language (or other bibliometric attributes) is not correctly assigned by WoS.

In this context, the alternating coverage of the original and the translated version of a given journal by WoS (but this time with the respective language correctly assigned) should be mentioned. For example, the papers of the original version of the Russian journal Zhurnal Eksperimentalnoi i Teoreticheskoi Fiziki (ZhETF), mentioned above, appear from 1945 to 1954 and from 1972 to 1996 as source items. Within the time period from 1955 to 1971, however, the translated version Soviet Physics JETP is covered. WoS coverage of another important Russian journal changed in a similar way: The original version of Uspekhi Fizicheskikh Nauk ranges from 1955 to 1964 and from 1972 to 2001. The translated version Soviet Physics Uspekhi fills the gap in between, ranging from 1965 to 1971 .

Another kind of database errors are called mapping errors, which can be defined as the failure to establish an electronic link between the cited references and the corresponding database records. A specific paper appearing as a reference in a source record (i.e., a paper covered by the database) will 
be linked with the corresponding full record, provided that this paper is also covered as a source record. Assuming that, for instance, a paper by Albert Einstein published in Annalen der Physik (a journal covered by WoS since 1900) refers to another paper published by this journal: The full record of the citing paper (the Einstein paper) will normally provide a link from the cited reference to the full record of the cited paper. There are different cases where this link is missing due to various kinds of mapping errors. For example, many references with Philosophical Magazine as the reference journal do not include volume and page numbers (e.g., E. Rutherford, Philosophical Magazine, May 1911). The references are not linked with the corresponding WoS source records and, hence, are not included in the citation counts given in the WoS records under "times cited."

\section{Translation Errors}

Translation errors of titles are a general problem of all literature databases and not only of the citation indexes. Again, historical papers are affected much more than current ones. At the beginning of the 20th century, many leading journals appeared in non-English languages (e.g., Annalen der Physik, Physikalische Zeitschrift, and Zeitschrift für Physik in the field of physics were published in German). For integration into literature databases and citation indexes, the titles of such publications are normally translated into English. However, the translation usually lacks higher standards and is mostly inconsistent.

A paper by the quantum physicist Max Born published in the journal Physikalische Zeitschrift (Born, 1914) is a typical example. The original German title was "Über die Methode der Eigenschwingungen in der Theorie der spezifischen Wärmen." In WoS, this title was translated as "Method of Natural Oscillations in the Specific Conduction Theory," which is clearly misleading. A more meaningful translation is given in the appropriate record of the field-specific INSPEC database: The title "Natural Vibrations and Specific Heats" provides far better information on the subject of the paper. This example stands for the numerous cases of deficient translation that can easily be found in papers with their titles translated into English. Note that translation errors of title words additionally imply limitations of the searching possibilities in literature searches.

\section{Misspelled Citations}

Misspelled citations (or references; the terms may be used interchangeably here) are a general problem in citation analysis and have been studied and discussed extensively (see Buchanan, 2006, and the references cited therein). The citations that refer to historical works, however, are particularly susceptible to "mutations." Citation errors are normally generated as transcription errors by the citing authors. In the course of time, erroneous references tend to mutate until the referenced work can barely be identified by the reader (Specht, 2010). The earlier the publication date of the cited papers, the higher the probability that reference mutations occur. If a reference to a historical paper is misspelled by a prominent author and/or a highly cited paper, then the percentage of erroneous citations possibly increases strongly (Cardona, Chamberlin, \& Marx, 2007). Another phenomenon has further increased the error rate of historical papers: In the past, authors frequently copied references and used them unchecked due to missing accessibility of the original papers (obviously, some authors still do not read the papers that they cite).

The WoS Cited Reference Search mode gives access to all references that appeared in source journal papers (cited correctly or erroneously). The alphabetical reference listing reveals all reference variants with regard to author names and the numerical data, including the reference journal names (journal titles). The journal name variants of the cited references result from the lack of standardization (with journal names not standardized by the citing authors or the citing journals or the database producer). The WoS citation counts given in the records under "times cited" comprise all errorfree citations with regard to the first author names and the numerical data. The various reference journal names of one and the same journal that appear in references do not matter. The citation counts result from matching only the first author and the numerical data of the references and not their journal names.

Analyzing the reference variants of current papers reveals that approximately $5 \%-10 \%$ of all citations are erroneous with respect to the numerical bibliographic data (i.e., volume number, starting page, and publication year). Errors in author names have not been taken into account here. They would add another $7 \%$ errors to the numerical data error rate according to an estimate given by Moed (2002). Errors in title words of the cited papers (if at all included in the references) are not relevant, because titles are not considered in citation matching. The estimated error rate of 5\%-10\% is only a rough order of magnitude, based on my experience and the various studies mentioned by Buchanan (2006). The error rates given in the various studies depend on the error definition and many other aspects, such as the specific journals and research fields analyzed.

In the case of historical papers, the percentage of misspelled citations is significantly higher. The increased number of citation errors in the case of older papers can be noticed easily in the WoS Cited Reference Search mode: through a comparison of the percentage of linked reference items (the electronic link between the cited references and the corresponding full records marked by "view record") with regard to earlier versus more recent reference publication years. For example, the reference item list with the reference journal Annalen der Physik and the reference publication year 1900 includes only $14 \%$ linked reference items, whereas the references of that same reference journal with the reference publication year 2000 includes $46 \%$ linked reference items.

Furthermore, we may also compare the citations of the ensembles of linked and nonlinked references of the same reference publication years 1900 and 2000. Provided that all 
TABLE 1. Total number of citations and the number and percentage of incorrect citations of some carefully analyzed individual papers. ${ }^{a}$

\begin{tabular}{lccr}
\hline Paper & $\begin{array}{c}\text { Total number of } \\
\text { citations (correct }+ \\
\text { incorrect) }\end{array}$ & $\begin{array}{c}\text { Number of } \\
\text { incorrect } \\
\text { citations }\end{array}$ & $\begin{array}{c}\text { Percentage } \\
\text { of incorrect } \\
\text { citations }\end{array}$ \\
\hline W. Kohn \& L.J. Sham (1965) & 19234 & $668^{\text {b }}$ & 3.47 \\
O.K. Andersen (1975) & 4562 & 89 & 1.95 \\
G. Mie (1908) & 4836 & 297 & 6.14 \\
R. Kohlrausch (1854) & 467 & 362 & 15.63 \\
J. Lindhard (1954) & 1945 & 177 & 18.61 \\
G. Wassermann (1933) & 216 & 81.94 \\
\hline
\end{tabular}

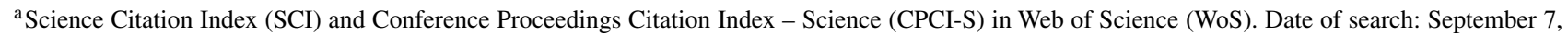
2010.

bIn addition, 1,648 citations appeared in the WoS Cited Reference Search mode with "J Phys Rev" instead of "Phys Rev" as the reference journal name. This is obviously caused by the fact that the reference standard of many journals is misleading here: "W. Kohn \& Sham L. J. Phys. Rev. 140 ..." (with no delimiter between the author name and the journal name), "J." can be taken as the initial for "journal" rather than as the second initial of the author's first name (which is L. J. Sham).

nonlinked references result from misspelled citations (this is only a rough approximation because of the possibility of mapping errors, see above), we obtain a fraction of $42 \%$ erroneous citations (here, with respect to either the author name or the numerical bibliographic data) for the Annalen der Physik references with the reference publication year 1900 and a fraction of $20 \%$ for the references with the reference publication year 2000. Hence, the error rate of the 1900 references is more than twice that of the 2000 references.

Several papers were found to be cited up to $80 \%$ with incorrect volume numbers and/or starting pages (i.e., the paper by Wassermann, 1933). Many references with early reference publication years can hardly be identified and assigned to specific papers. Consequently, citation counting of historical papers could become highly problematic. In Table 1, the total number of citations as well as the number and percentage of incorrect citations of some carefully analyzed more recent and historical papers are given.

Recently, J. K. Wetterer examined "how quotation error (misrepresentation of previous work) and citation copying (citing unexamined publications referred to by others) led to the origin and spread of the erroneous story of ant extinctions in Madeira" (Wetterer, 2006, p. 351). With regard to historical papers, Wetterer makes an interesting statement: "If quotation error and citation copying are common when citing easily accessible, recent publications, these problems are probably even more common when authors cite old references that are difficult to acquire or written in foreign languages. Papers written in foreign languages may be mistranslated and are probably more likely to be left unread" (p. 364).

\section{Complex Author Names}

The transcription of author names, another common problem in literature databases and citation indexes, is particularly relevant for citation searching of historical papers. Complex author names seem to be more common among authors of historical literature. Therefore, considering the different kinds of spelling is exceedingly important to ensure completeness. For example, the reference author name of Carl von Linné, one of the most frequently cited early scientists (Marx \& Cardona, 2004), may appear in the WoS Cited Reference Search mode as "vonLinne C" but also in Latin as "vonLinnaeus C" (or sometimes as "vonLinneus C"). Authors that have the title of Lord may be listed under their titles or under their names (e.g., Lord Kelvin alias William Thomson or Lord Rayleigh alias John William Strutt).

The WoS specific notation of complex surnames is inconsistent, with respect to both the author names (appearing in the database records) and the reference author names (appearing in the cited references). The database records from 1900 to 1944 and from 1998 to present divide complex author surnames into separate terms (e.g., "von Laue M"), whereas from 1945 to 1997, complex surnames appear in one word (e.g., "vonLaue M"). The reference items in the Cited Reference Search mode with reference publication years before 1998 generally express complex surnames in one word (e.g., "vonLaue M" rather than "von Laue M"). Such names may also be cited in the shortened version (e.g., "Laue M").

\section{Complicated Journal Names}

Much highly relevant historical literature is not covered by the WoS source journals (see above). Nevertheless, the citations of such papers are searchable via the WoS Cited Reference Search mode, because the cited references are not limited to papers published in source journals. They include any other published material, in particular, papers not published in source journals and book references. Some nonsource journals have rather complicated non-English journal names, such as Verhandlungen der Deutschen Physikalischen Gesellschaft (see the famous papers by Max Planck (1900a,b) on the quantization of energy) or Matematisk-Fysiske Meddelelser Kongelige Danske Videnskabernes Selskab (the journal in which the paper by Lindhard, 1954, was published). In contrast to journals like Physical Review, the journal names of such complicated journals are hardly manageable and difficult to allocate. Because of the lack of standardization, the journal name variants of the cited references are already numerous. Complicated journal names further increase the 
overall number of reference variants appearing in the Cited Reference Search mode.

Many long-lived journals changed their journal names one or more times in the course of time. For example, if the citations of the papers published in the journal Annalen der Physik (in particular the pre-1900 papers not covered as WoS source journal records) have to be analyzed based on the WoS Cited Reference Search mode, then the following journal names (abbreviated or in full or any mixture of both) in combination with the relevant reference publication years (given in parentheses) have to be taken into consideration:

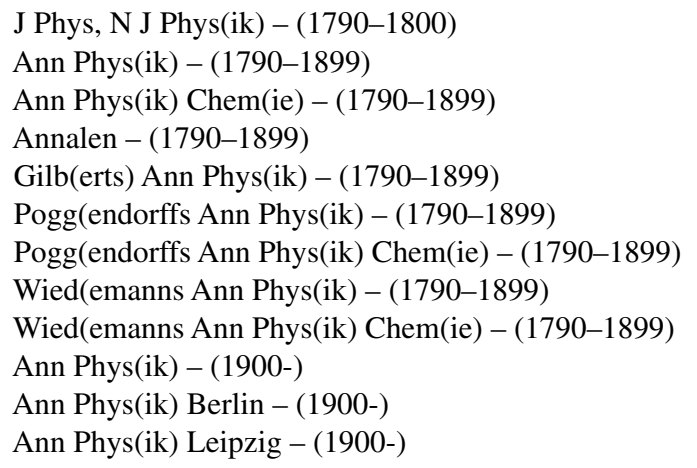

\section{Additional Sources of Errors}

In addition to the problems mentioned so far, there are several specific sources of errors resulting from the citation practice at the beginning of modern science. The original paper by Rudolf Kohlrausch (1854) on the stretched exponential function provides a good example of 19th century publication practices that are highly uncommon for presentday papers and are mostly forgotten today. Following the policy of Annalen der Physik (and also of other journals) at that time, the initial part of some papers was placed at the beginning of an issue and then continued later on, with work by other authors placed in between. Within the citations of the paper by Kohlrausch (1854), the starting page 179 is the page number most commonly found in the current literature. However, it is the starting page of the second part of the paper where Kohlrausch introduced the concept of the stretched exponential function-the actual starting page of the full paper is page 56. This kind of complex pagination complicates the assignment of references to specific papers when performing the Cited Reference Search in WoS and adds further confusion to that discussed so far. Note also that in historical papers the figures sometimes appeared on separate pages and not within the range of the text pages (but mostly at the end of the relevant journal issues).

For many present-day scientists non-English 19th century journals like Annalen der Physik are difficult to acquire and to read. Sometimes early papers are confused and the confusion is possibly not rectified until decades after the errors were introduced, if at all. Again, the famous paper by Kohlrausch mentioned above is a good example. Until present, most citing authors do not refer to the correct paper (Kohlrausch, 1854), referring instead to an earlier paper (Kohlrausch, 1847) in which he only qualitatively described relaxation phenomena (Cardona et al., 2007). A quite similar confusion of historical papers was discussed concerning the first report on exfoliated graphite (Boehm \& Stumpp, 2007). Occasionally, author names have been confused, and prominent researchers have been cited instead of their students (e.g., Ostwald, 1900, rather than Hulett, 1901). Errors of this kind are hardly found systematically and are usually discovered by chance. The Ostwald/Hulett confusion, for example, was discovered by Wolfgang Hönle (Hönle, 2004), who kindly notified me.

\section{Time-Adjustment of Citation Counts}

The number of publications per year published in the field of physics increased approximately by a factor of hundred throughout the 20th century. The time evolution of the physics literature shows a remarkable increase in the number of items per year, starting between 1955 and 1960. It is easy to conjecture that this bend was due to what is today called the Sputnik shock (Dickson, 2001), which caused a rapid increase in research activities. Consequently, the number of scientists and publications also increased dramatically. Following the title of the famous book by Derek de Solla Price (1963), we may speak of the period before 1955 as "little science," whereas the period after 1960 can be referred to as "big science." The number of publications of the little science period was not only low but the publications were also cited less frequently than those of the big science period. This is clearly reflected in the low average number of citations per publication as compared with the present average number. In fact, the probability of a paper being cited depends on the average number of references it musters.

Because WoS extends back to 1900 , not only the papers from the first half of the 20th century but also their citations are available for bibliometric investigations. The question emerges as to how the citation impact of these papers may be evaluated in comparison with the impact of contemporary papers. This requires either standardizing the citation rates with the help of reference standards or readjusting them to suit the present practice within the field. For example, the citation counts of papers written by the pioneers of science given under "times cited" in the WoS can only be compared with the citation counts for papers by contemporary scientists, if the relationship between citation counts of earlier and contemporary papers can be ascertained.

The procedure for time standardization and time adjustment suggested by Marx, Bornmann, and Cardona (2010) ought to be of interest especially for studies in the history of science. In that discipline, the aim is often to examine early pioneers in the sciences and their lifework (usually highly recognized scientists, such as early Nobel laureates). Bibliometrics offers quantitative data on the scientists and their work in the form of time-dependent citation counts (citation history). If the citation history extends over the boundary year of 1960, dividing the eras of little science and big science, 
the schemas offered should facilitate a better interpretation of the impact data.

\section{Informal Citations and Obliteration}

The fundamental ideas of science soon find their way into textbooks. Once they are then taken for granted, scientists usually no longer refer to the original papers. The incorporation of information is a natural process in science, and the seminal knowledge is referred to only if special viewpoints demand it. In that case, seminal work is mostly cited by mentioning the author's name or name-based items ("informal citations," also called eponyms, see Marx \& Cardona, 2009) instead of citing the full references as a footnote ("formal citations"). We should bear in mind that this specific bias may lead in some cases to a substantial loss of the citation-based impact, in particular in the case of historical papers.

Another important aspect concerning citation counts of historical papers is "obliteration by incorporation," a phenomenon first described in 1949 by the sociologist Robert K. Merton (1949, 1965). The process of obliteration usually affects seminal works (i.e., truly ground-breaking research), offering novel ideas that are rapidly absorbed into the body of scientific knowledge. Seminal work is soon integrated into textbooks and becomes increasingly familiar within the scientific community. Because of this absorption and canonization, the original sources fail to be cited, either as full references (formal citations) or even as names or subjectspecific terms (informal citations). Hence, formal or even informal citation counts of seminal papers cannot be taken as a measure of their influence in modern science. There is no metric for quantifying fundamentality, significance, or even elegance, which are terms that fall under a completely different category.

\section{Conclusions}

This compilation of some special features of historical works relevant for literature and citation retrieval provides some hints on avoiding fatal errors when searching this kind of literature. The most relevant pitfalls and problems are discussed and demonstrated based on typical examples. The integration of historical works into current papers as well as studies in the history of science both deserve accurate and careful usage of the available databases, in particular, the WoS Century of Science backfiles. The issues presented and discussed here have been explored and applied in connection with various studies on the posthumous impact of historical scientists (Cardona \& Marx, 2006a,b, 2008a,b; Cardona et al., 2007; Marx \& Cardona, 2006, 2009) and on other topics related to history. Recently, we appraised Kuhn's theoretical approach by conducting a historical reconstruction and a citation analysis, taking as a case study the transition from the static view of the universe to the Big Bang theory in cosmology (Marx \& Bornmann, 2010). In consideration of further upcoming archives and their potential usefulness for studies in the history of science, it makes sense to address this special aspect of bibliometrics.

\section{Acknowledgement}

The author expresses his sincere gratitude to Professor Wieslaw Wojnowski (Chemical Department, Technical University of Gdańsk, Poland) for early encouragement to use databases for exploring the history and sociology of science.

\section{References}

Andersen, O.K. (1975). Linear methods in band theory. Physical Review B, 12(8), 3060-3083.

Boehm, H.P., \& Stumpp, E. (2007). Citation errors concerning the first report on exfoliated graphite. Carbon, 45(7), 1381-1383.

Born, M. (1914). Über die Methode der Eigenschwingungen in der Theorie der spezifischen Wärmen [Natural vibrations and specific heats]. Physikalische Zeitschrift 15, 185-191.

Buchanan, R.A. (2006). Accuracy of cited references: The role of citation databases. College \& Research Libraries, 67(4), 292-303.

Cardona, M., Chamberlin, R.V., \& Marx, W. (2007). Comment on the history of the stretched exponential function. Annalen der Physik, 16(12), 842-845. DOI: 10.1002/andp.200710269

Cardona, M., \& Marx, W. (2006a). Vitaly L. Ginzburg - A bibliometric study. Journal of Superconductivity and Novel Magnetism, 19(3-5), 459-466. See also an updated version in Ginzburg, V.L. (2009). On superconductivity and superfluidity - A scientific autobiography. Springer, Berlin Heidelberg. DOI: 10.1007/s10948-006-0173-8

Cardona, M., \& Marx, W. (2006b). The posthumous impact of Paul Drude. Annalen der Physik, 15(7-8), 461-468. DOI: 10.1002/andp.200510196

Cardona, M., \& Marx, W. (2008a). Max Planck - A conservative revolutionary. Il Nuovo Saggiatore, 24(5-6), 39-54.

Cardona, M., \& Marx, W. (2008b). Max Born and his legacy to condensed matter physics. Annalen der Physik, 17(7), 497-518. DOI: 10.1002/andp.200810304

de Solla Price, D.J. (1963). Little science, big science. New York: Columbia University Press.

Dickson, P. (2001). Sputnik: The shock of the century. New York: Walker \& Co.

Hönle, W. (2004). Über das Zitieren [On citing behavior]. Mitteilungen der Wilhelm-Ostwald-Gesellschaft Großbothen/Sachsen, 9(4), 60-61.

Hulett, G.A. (1901). Beziehungen zwischen Oberflächenspannung und Löslichkeit [Relations between surface-tension and solubility]. Zeitschrift für Physikalische Chemie, 37(4), 385-406.

Kohlrausch, R. (1847). Über das Dellmann'sche Elektrometer [About Dellman's electrometer]. Poggendorff's Annalen der Physik und Chemie $72,353-405$.

Kohlrausch, R. (1854). Theorie des elektrischen Rückstandes in der Leidener Flasche [Theory of electric residue in the Leyden jar]. Poggendorff's Annalen der Physik und Chemie 91, 56-82, 179-214.

Kohn, W., \& Sham, L.J. (1965). Self-consistent equations including exchange and correlation effects. Physical Review, 140(4A), 1133-1138.

Lindhard, J. (1954). On the properties of a gas of charged particles. Matematisk-Fysiske Meddelelser Kongelige Danske Videnskabernes Selskab, 28(8), 1-57.

Marx, W. (2001). Angewandte Chemie in light of the Science Citation Index. Angewandte Chemie - International Edition, 40(1), 139-143. Die Angewandte Chemie im Lichte des Science Citation Index. Angewandte Chemie, 113(1), 143-148. DOI: 10.1002/15213773(20010105)40:1<139::AID-ANIE139>3.0.CO;2-4

Marx, W. (2009). The anatomy of the International Journal of Materials Research in the light of bibliometrics. International Journal of Materials Research, 100(1), 11-23. DOI: 10.3139/146.101793

Marx, W., \& Bornmann, L. (2010). How accurately does Thomas Kuhn's model of paradigm change describe the transition from a static to a 
dynamic universe in cosmology? A historical reconstruction and citation analysis. Scientometrics, 84(2), 441-464. DOI: 10.1007/s11192-0090107-X

Marx, W., Bornmann, L., \& Cardona, M. (2010). Reference standards and reference multipliers for the comparison of the citation impact of papers published in different time periods. Journal of the American Society for Information Science and Technology, 61(10), 2061-2069. DOI: 10.1002/asi.21377

Marx, W., \& Cardona, M. (2004). Blasts from the past. Physics World, 17(2), $14-15$.

Marx, W., \& Cardona, M. (2009). The citation impact outside references Formal versus informal citations. Scientometrics, 80(1), 1-21. DOI: 10.1007/s11192-008-1824-2

Merton, R.K. (1968, first edition: 1949). Social theory and social structure. New York: The Free Press.

Merton, R.K. (1965). On the shoulders of giants: A Shandean postscript. New York: The Free Press.

Mie, G. (1908). Beiträge zur Optik trüber Medien, Metalllösungen [Contributions to the optics of turbid media, especially colloidal metal solutions]. Annalen der Physik, 25(3), 377-445.

Moed, H.F. (2002). The impact-factors debate: The ISI's uses and limits. Nature, 415(6873), 731-732.

Ostwald, W. (1900). Über die vermeintliche Isomerie des roten und gelben Quecksilberoxyds und die Oberflächenspannung fester Körper [On the supposed isomerism of red and yellow mercury oxide and the surfacetension of solid substances]. Zeitschrift für Physikalische Chemie, 34(4), 495-503.

Planck, M. (1900a). Über eine Verbesserung der Spektralgleichungen [On an improvement of Wien's equation for the spectrum]. Verhandlungen der Deutschen Physikalischen Gesellschaft, 202-204.

Planck, M. (1900b). Zur Theorie des Gesetzes der Energieverteilung im Normalspektrum [On the theory of the law on energy distribution in the normal spectrum]. Verhandlungen der Deutschen Physikalischen Gesellschaft, 237-245.

Pratt, S.M., \& Stembridge, R. (2006, September). An analysis of citations in scientific and patent literature to historical research from the first half of the 20th century and the relationship to the accessibility of these works on electronic archives. Paper presented at the CINF Symposium, American Chemical Society 232nd National Meeting 2006, San Francisco, CA.

Specht, C.G. (2010). Opinion: Mutations of citations. Retrieved from http://www.the-scientist.com/news/display/57689/

Wassermann, G. (1933). Einfluss der alpha-gamma-Umwandlung eines irreversiblen Nickelstahls auf Kristallorientierung und Zugfestigkeit [Influence of the alpha-gamma transformation of an irreversible nickel steel on crystal orientation and tensile strength]. Archiv für das Eisenhüttenwesen, 6(8), 347-351.

Wetterer, J.K. (2006). Quotation error, citation copying, and ant extinctions in Madeira. Scientometrics, 67(3), 351-372. 